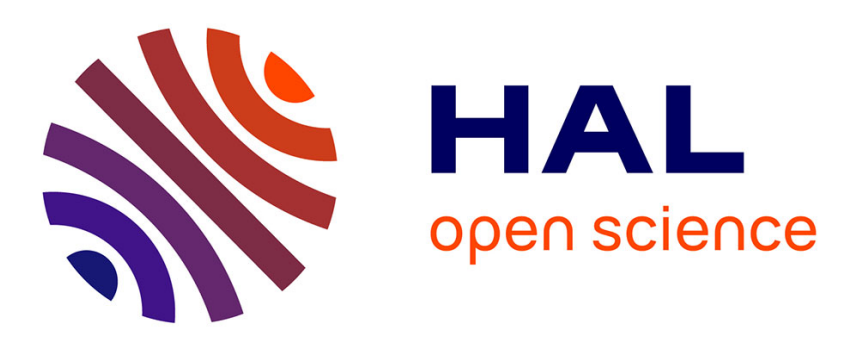

\title{
First principles calculations of the electronic structures of magnetic compounds
}

Samir F. Matar

\section{To cite this version:}

Samir F. Matar. First principles calculations of the electronic structures of magnetic compounds. Journal de Physique IV Proceedings, 1997, 07 (C1), pp.C1-169-C1-172. 10.1051/jp4:1997161 . jpa00255108

\section{HAL Id: jpa-00255108 https://hal.science/jpa-00255108}

Submitted on 1 Jan 1997

HAL is a multi-disciplinary open access archive for the deposit and dissemination of scientific research documents, whether they are published or not. The documents may come from teaching and research institutions in France or abroad, or from public or private research centers.
L'archive ouverte pluridisciplinaire HAL, est destinée au dépôt et à la diffusion de documents scientifiques de niveau recherche, publiés ou non, émanant des établissements d'enseignement et de recherche français ou étrangers, des laboratoires publics ou privés. 


\title{
First Principles Calculations of the Electronic Structures of Magnetic Compounds
}

\author{
S.F. Matar \\ Institut de Chimie de la Matière Condensée de Bordeaux, ICMCB-CNRS, Château Brivazac, avenue du \\ Docteur Albert Schweitzer, 33608 Pessac cedex, France
}

\begin{abstract}
Modem methods for the calculation of the band structure of magnetic materials are based on the density functional theory where the effects of exchange and correlation are treated within the local density approximation. In this framework the description of the physical properties of a large number of compounds ranging from metallic alloys to several oxide systems gives satisfactory results in a majority of cases. More specifically, properties of magnetic materials can be obtained such as the saturation magnetization (spin only and orbital moments), the magneto-volume effects $E(V, M)$, the Fermi conctact term of the hyperfine field $\left(H_{F C}\right)$, the magnetocrystalline anisotropy $\xi$ and to a certain extent the magnetic order temperatures $\left(T_{C}, T_{N}\right)$. Moreover non-collinear magnetic structures can now be calculated. In this work a non-exhaustive description of the basics of the theory will be provided and followed with illustrative examples.
\end{abstract}

\section{Introduction.}

Historically, within the Born-Oppenheimer adiabatic approximation which consists of treating the motions of the electrons separately from those of the nuclei, the first solution to the single electron problem is known as the Hartree-Fock (HF) method. The intractable $N$-elecron system is approached by a determinant of single electron wave functions. However the resulting wave function is not ensured to be orthogonal. Fock introduced then a further term to treat exchange. It plays a role of reducing electrostatic interaction and accounts for the fact that two electrons with the same spin cannot approach each other indefinitely. This is exact except that a further contribution is missing which is relative to correlation. According to $\mathrm{HF}$ method no correlation exists for the trajectories of electrons with opposite spins; these are then allowed to be in the same region of space. This leads to neglect a « correlation hole " around the electron which is best visualized as an impenetrable space surrounding it. Thus the energy associated with correlation appears as the difference between the total energy of the $N$ electron system and HF energy. Although improvements on $\mathrm{HF}$ to include this correlation were attempted leading to tedious and extremely time consuming computations, a major alternative was brought in 1964 by the density functional theory (DFT). In shorthand presentation, instead of conducting the variational procedure on the wave function in HF (multi Slater determinants), it is a functional of the electron density $F[\rho(\mathrm{r})]$ which becomes the variational variable in the DFT. Exchange and correlation (XC) are equally treated at a local level within the local density approximation (LDA) as it will be shown. This work is organized as follows : next section is devoted to a presentation of the DFT-LDA formalism and to a short discussion of band magnetism. This will be followed by illustrative examples.

\section{Conceptual framework and some formalism.}

2.1. Density functional theory.

Due to its extended use since its development by Hohenberg and Kohn (HK) [1] then by Kohn and Sham (KS) [2], several articles have been devoted to the mathematical presentation of the DFT [see 3 for a review]. It is not our purpose here to give an exhaustive account of this theory but only to present those details of interest to our presentation.

HK demonstrated two theorems which can be formulated as follows : the total energy of the system is a unique functional of the electron density; its minimum corresponds to the energy of the ground state (we implicitly calculate $\mathrm{OK}$ properties of the system). KS then expressed the total energy functional for an interacting electron gas in a static potential $v(r)$ as follows :

$$
E[\rho(\mathbf{r})]=F[\rho(\mathbf{r})]+\int \rho(\mathbf{r}) v(\mathbf{r}) d^{3} \mathbf{r} ;
$$


where : $\quad F[\rho(\mathbf{r})]=T[\rho(\mathbf{r})]+1 / 2 \iint \rho(\mathbf{r}) \rho\left(\mathbf{r}^{\prime}\right) /\left|\mathbf{r}-\mathbf{r}^{\prime}\right| \mathbf{d}^{3} \mathbf{r} \mathbf{d}^{3} \mathbf{r}^{\prime}+E_{x c}[\rho(\mathbf{r})]$

is a universal functional (unknown because of the right hand side third quantity) whose terms are relative to kinetic energy $(T[\rho(r)])$, Hartree interaction energy $\left(1 / 2 \iint \rho(\mathbf{r}) \rho\left(\mathbf{r}^{\prime}\right) /\left|\mathbf{r}^{\prime}-\mathbf{r}^{\prime}\right| d^{3} \mathbf{r} d^{3} \mathbf{r}^{\prime}\right)$ and exchange-correlation (XC) energy ( $\left.\mathrm{E}_{\mathrm{xc}}[\rho(\mathbf{r})]\right)$. Hence $\mathrm{XC}$ term appears as the difference between the true kinetic energy and that of a non-interacting electron gas system + the difference between the true interaction energy and that of Hartree. It represents our ignorance of the actual system and its accurate evaluation is the cornerstone of modern computational methods.

\subsection{The local density approximation (LDA) to the DFT.}

In a slowly varying electron density, $K S$ proposed a development for $E_{x c}[\rho(r)]$ :

$$
E_{x c}[\rho(\mathbf{r})]=\int \rho \varepsilon_{x c}(\rho) d^{3} \mathbf{r}+\int|\nabla \rho|^{2} \varepsilon_{x c}(\rho) d^{3} \mathbf{r}+
$$

The LDA consists of asssuming that $\mathrm{E}_{\mathrm{xc}}[\rho(\mathbf{r})]$ is a local functional of the electron density $\rho(\mathbf{r})$, i.e., it depends on the density at $\mathbf{r}$. The gradient term $\nabla \rho(\mathbf{r})$ as well as higher order terms are then omitted. Thus $E_{x c}[\rho(\mathbf{r})]=\int \rho \varepsilon_{x c}(\rho) d^{3} r$ where $\varepsilon_{x c}$ is the contribution of exchange and correlation to the total energy per electron in a homogeneous, but interacting electron gas. Note here that $\varepsilon_{x c}$ is a function of electron density. Different parameterization schemes exist for $\varepsilon_{x c}$ such as that of Hedin and Lundqvist [4] and that of von Barth and Hedin [5]. They lead to a high numerical accuracy of $\varepsilon_{x c}$ which makes $E_{x c}[\rho(r)]$ the principal approximation of the calculations undertaken in the framework of this theory. The corresponding XC potential $\mathrm{V}_{\mathrm{xc}}(\mathbf{r})=\delta \mathrm{E}_{\mathrm{xc}} / \delta \rho(\mathbf{r})$ is included into the overwhole potential as follows :

$$
V(\mathbf{r})=V_{\text {ions }}(\mathbf{r})+V_{\text {eff }}(\mathbf{r}) \text { with } V_{\text {eff }}(\mathbf{r})=\int_{\rho}\left(\mathbf{r}^{\prime}\right) /\left|\mathbf{r}_{-} \mathbf{r}^{\prime}\right|+V_{x c}(\mathbf{r}) \text {. }
$$

A generalization of the LDA to spin polarized cases consists of assuming $\rho \uparrow$ and $\rho \downarrow$ in the density matrix and writing the $\operatorname{spin}(\alpha)$ dependent XC potential : $\mathrm{V}_{\mathrm{xc}}^{\alpha}(\mathbf{r})(\alpha=\uparrow$ or $\downarrow)$ where $\varepsilon_{\mathrm{xc}}$ is now $\varepsilon_{\mathrm{xc}}(\rho \uparrow, \rho \downarrow)$.

The wave equation shows then an explicit dependence on the spin of the wave functions $\psi$ and of the potential $\mathrm{V}$ :

$$
\left\{-\nabla^{2}+V^{\alpha}(\mathbf{r})-E_{\alpha k}\right\} \psi_{\alpha k}(\mathbf{r})=0(\mathbf{k}: \text { wave vector }) \text {. }
$$

This wave equation is referred to as Kohn-Sham equation. The single-particle HF and KS equations do not have the same physical meaning! In the latter we are dealing with a quasi-particle, i.e., the electron and its correlation hole. It should be stressed that the calculations are $a b$ initio: One starts from the neutral atomic configurations (valence states explicitly) which only serve as a first guess of the crystal electron density. The procedure is then self-consistently carried out because the potential and the wave functions are interdependent. The variational solution of the problem is made easier in linearized methods, such as the augmented spherical wave (ASW) [6] and the linearized augmented plane wave (LAPW) [7] methods. The energy dependence of the wave function $\psi_{\mathrm{kL}}(\mathrm{r})$ ( $\mathrm{L}: l$ and $\mathrm{m}$ quantum numbers) is lifted and the variational procedure conducted on the energy dependent coefficients $c_{\mathrm{kL}}$ in $\psi_{\mathrm{kL}}(\mathbf{r})=\mathrm{c}_{\mathrm{kL}} \chi_{\mathrm{I}}(\mathbf{r})$. KS did not expect the LDA -LSDA- to be efficient in describing real chemical systems where the density does not vary slowly between the sites. However in a majority of cases (see following sections) results in good agreement with experiment are obtained. As to why this is so, the question is still an open one. Nevertheless the shortcomings to this approximation such as overestimated binding energies and too small energy gaps for insulating materials led to try improvements on the LSDA. The GGA (generalized gradient approximation) [8], where gradient terms such as in the one in the above development of $E_{x c}[\rho(r)]$ are included, is one of them. Better results are observed for instance for the band gaps and band splittings in transition metal oxides and fluorides [9]. However no systematic improvements on the LSDA could be traced out especially when dealing with heavy elements like Pd or with the magnetic properties which are overestimated [9]. Another attempt to improve on the LSDA is the LSDA+U correction brought about to account for the insulating character of transition elements monoxides, ex. NiO. Within the formalism above (mean field, central potentials) the d-orbitals are equally occupied (in principle ; see examples) whereas in physical reality they are not due to crystal field effects. This is corrected by an extra term added to the LSDA potential for every specific orbital using an adjustable Coulomb interaction parameter $U$ to obtain the correct gap [10].

\subsection{Band magnetism.}

The explicit spin-dependence of the wave equation leads to a solution for each spin direction independently. One usually starts with a charge imbalance for each atomic species, i.e. with a non-zero magnetic moment defined as $m=n \uparrow-n \downarrow$ and $Z_{v}$ $=\mathbf{n} \uparrow+\mathbf{n} \downarrow\left(Z_{v}\right.$ is the number of valence electrons). Now $n_{\alpha}=\int_{v} \Sigma_{k}\left|\psi_{\alpha k}(r)\right|^{2} d^{3} r$ is the integrated charge density ; the sum extends over lower states filled up to a common Fermi energy $E_{F}$ for both spin populations. Self-consistency can either lead to $m=0$ or to finite magnetic moments which are generally found in good agreement with experiment. When a stable magnetic state is found the band structures are different for the two spin directions. From this one can define two quantities $<\Delta \mathrm{E}>$, the energy difference between $\uparrow$ and $\downarrow$ spin populations, called exchange splitting -most significant for $\mathrm{d}$ and $\mathrm{f}$ bands- and $\mathrm{I}$, exchange-correlation integral [11] such that $\langle\Delta \mathrm{E}\rangle=\mathrm{I}$.m. Despite this usual procedure to self-consistently calculate magnetic systems, it is enlightening to start from a non-magnetic configuration (total spins, $Z_{v}$ ) and ask about the conditions to be met for the occurrence of a magnetic moment. If one starts by creating a slight imbalance of the spin density, a small moment $\mathrm{m}$ appears. This involves a spin flipping whereby the $\uparrow$ population increases and the $\downarrow$ one decreases. This causes kinetic energy to rise by $1 / 2 \mathrm{~m} / n\left(\mathrm{E}_{\mathrm{F}}\right)\left(n\left(\mathrm{E}_{\mathrm{F}}\right)\right.$ : non-magnetic density of states at Fermi energy) and there is a gain in exchange energy of $-1 / 2$ $\mathrm{Im}^{2}$. The total energy counted from the non-magnetic state then amounts to: $\mathrm{E}=1 / 2\left\{\mathrm{~m}^{2} / n\left(\mathrm{E}_{\mathrm{F}}\right)\right\}\left\{1-1 / \mathrm{I} n\left(\mathrm{E}_{\mathrm{F}}\right)\right\}$. If $\mathrm{In}\left(\mathrm{E}_{\mathrm{F}}\right) \geq 1$ the system is unstable in a non-magnetic configuration and a magnetic moment should develop via intra-band polarization. This 
is the well known Stoner criterion for band ferromagnetism [12,13]. Ferromagnetism is implicit here because we are dealing with one site at a time. This is different from long range magnetic orders (ferro-, ferri-, antiferro- or even spin spirals) which can be hierarchized through total energy minima.

\section{Ilustrative examples.}

3.1 A half metallic ferromagnet: $\mathrm{CrO}_{2}$,

Chromium dioxide, one of the rare ferromagnetic oxides at room temperature $\left(T_{C}=398 \mathrm{~K}\right)$, has incited many investigations since its discovery such as in the domain of high density magnetic recording and the substitution of $\mathrm{Cr}$ by Ir leading to a large enhancement of the magnetocrystalline anisotropy [14]. According to the phenomenological description of its band structure by J.B. Goodenough, the $2 \mu_{\mathrm{B}}$ magnetic moment of $\operatorname{Cr}(\mathrm{IV}),\left(3 \mathrm{~d}^{2}\right)$ is due to one spin localized in a $t_{2 g}$ orbital and to another one, delocalized but strongly correlated in a narrow band formed of the other two $t_{2 g}$ orbitals and crossed by $E_{F}[15]$. Our investigation of the electronic and magnetic structure of $\mathrm{CrO}_{2}$ [16] was done in the framework of the above described theory using the augmented spherical wave (ASW) method. It gives an alternative description of the band structure and proposes a new physical property. Figure 1 gives the total density of states (DOS) of $\mathrm{CrO}_{2}$ for the majority $(\uparrow)$ and minority $(\downarrow)$ spin directions. The states can be assigned as follows with increasing energy : $O(2 s)$ around $-18 \mathrm{eV}, O(2 p)$ centered on $-5 \mathrm{eV}$ and $\mathrm{Cr}(3 \mathrm{~d})$. The latter states are splitted by the oxygen crystal field in the nearly regular

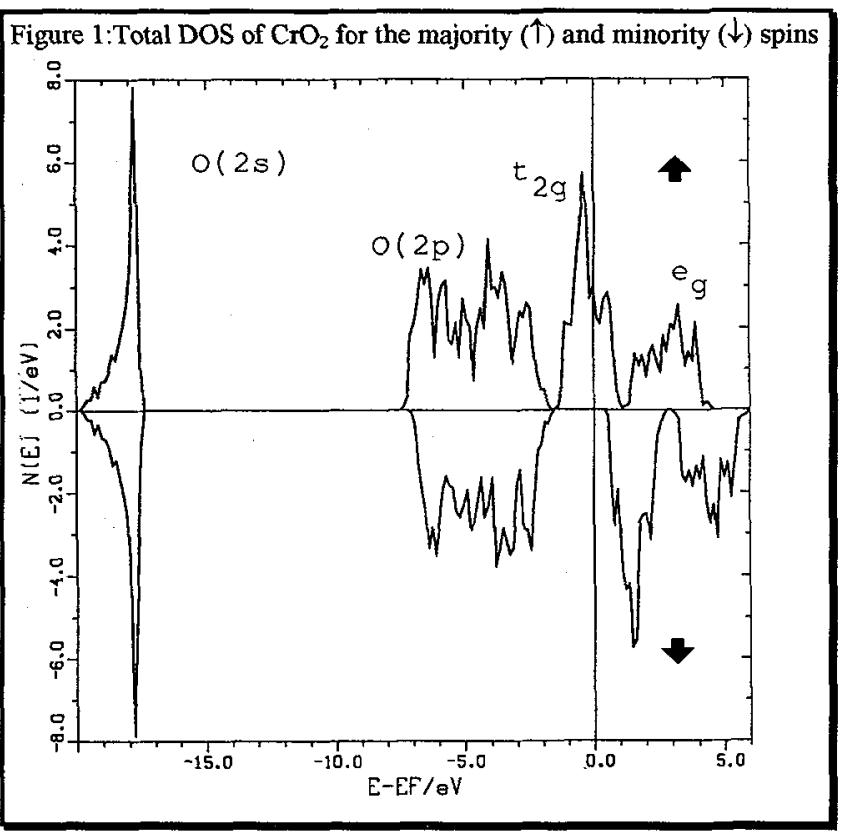

$\mathrm{CrO}_{6}$ octahedron into $\mathrm{t}_{2 \mathrm{~g}}$ (around $\mathrm{E}_{\mathrm{F}}$ ) and $\mathrm{e}_{\mathrm{g}}$ (above $\mathrm{E}_{\mathrm{F}}$, thus empty for $\uparrow$ and $\downarrow$ spin DOS). It is interesting to notice that the crossing of $E_{F}$ of a narrow $t_{2 g}(\uparrow)$ band $\left(t_{2 g}(\downarrow)\right.$ empty) and the presence of a sharp localized peak in this band, resemble the phenomenological band scheme. But $\mathrm{E}_{\mathrm{F}}$ falls in $\mathrm{a} \approx 2 \mathrm{eV}$ gap in minority spin band. Thus the integer $2 \mu_{\mathrm{B}}$ magnetic moment arises from this property of a $100 \%$ spin polarization in majority spin band. This leads to a predicted half metallic ferromagnet property for $\mathrm{CrO}_{2}$ which was experimentally verified a posteriori by magneto-optical spectroscopy [17].

\subsection{The « antiferromagnet $» \mathrm{SrFeO}_{3}$.}

For this metallic oxide crystallizing in the simple cubic perovskite structure $\mathrm{ABX}_{3}$, neutron diffraction investigation [18] propose a helical antiferromagnetic (AF) structure with a small magnitude propagation vector along the $\langle 111\rangle$ direction and $\mathrm{T}_{\mathrm{N}}=134 \mathrm{~K}$. The magnetic moment carried by $\mathrm{Fe}$ of $\approx 3.1 \mu_{\mathrm{B}}$ is accompanied by a large magnitude $\mathrm{O}$ moment of $\approx 0.3 \mu_{\mathrm{B}}$. Our investigation of the electronic and magnetic structure of $\mathrm{SrFeO}_{3}$ assumes different magnetic configurations (including an $\mathrm{AF}$ ordering along the $\mathrm{c}$ axis) which are calculated with identical convergence criteria and $\mathbf{k}$-point mesh in the Brillouin zone to enable for comparisons [19]. Next table summarizes the results of total energies (energy reference at -9341 Ryd.) as well as their differences between the different magnetic configurations (non magnetic (NM) (total spins, no spin polarization), feromagnetic $(\mathrm{F})$ and two $\mathrm{AF}$ orderings along the $\mathrm{z}$ axis and the cube diagonal respectively). The magnetic moments carried by $\mathrm{Fe}$ and $\mathrm{O}$ as well as the corresponding Fermi contact $(\mathrm{FC})$ hyperfine fields in Tesla $(\mathrm{T})$ are given too.

\begin{tabular}{|l|l|l|l|l|l|}
\hline Calculation & Tot. Ene. (Ryd.) & $\Delta \mathrm{E}(\mathrm{mRyd})$ & $\mathrm{M}_{\mathrm{Fe}}\left(\mu_{\mathrm{B}}\right)$ & $\mathrm{M}_{\mathrm{O}}\left(\mu_{\mathrm{B}}\right)$ & $\mathrm{H}_{\mathrm{FC}(\mathrm{Fe})} / \mathrm{H}_{\mathrm{FC}(\mathrm{O})}(\mathrm{T})$ \\
\hline Non magnetic & -0.455036 & $/$ & $/$ & $/$ & $/$ \\
\hline Ferromagnetic & -0.512835 & -57.799 & 2.964 & 0.152 & $-24.82 / 3.76$ \\
\hline Antiferro-<001> & -0.507830 & -52.794 & 3.005 & 0.138 & $-28.23 / 5.31$ \\
\hline Antiferro-<111 & -0.496371 & -41.335 & 2.970 & $/$ & -32.21 \\
\hline
\end{tabular}

The increase of $\mathrm{H}_{\mathrm{FC}}$ from the $\mathrm{F}$ to $\mathrm{AF}$ configurations follows that of $\mathrm{Fe}$ and $\mathrm{O} n \mathrm{~s}(n=1,2,3,4)$ electron densities at the nucleus polarized by the $d$ moment. For $\mathrm{Fe}, \mathrm{H}_{\mathrm{FC}}(\mathrm{AF})$ are close to $30 \mathrm{~T}$ in fair agreement with experimental findings for tetravalent iron although our calculations are far from giving such an electronic configuration. Whereas a moment carried by oxygen is evidenced, it has a twice smaller magnitude than that experimentally proposed. The magnitude of the moment 
carried by $\mathrm{Fe}$ is in agreement with experiment, especially for the AF $<001>$ configuration. We can analyse it from the charge distribution over $t_{2 \mathrm{~g}}$ and $\mathrm{e}_{\mathrm{g}}$ manifolds in the octahedral crystal field of oxygen atoms by introducing the spherical harmonics for the $5 \mathrm{~d}$ orbitals. Considering the first value for instance $(F)$, one gets the following occupations $\left(e=\right.$ electron) : $t_{2 g}(\uparrow)=$ 2.88 é, $e_{g}(\uparrow)=1.37$ é, $t_{2 g}(\downarrow)=0.71$ é and $e_{g}(\downarrow)=0.58$ é. The total $t_{2 g}$ population is then 3.59 é, i.e. more than half filled manifold whereas the 1.95 é for $e_{g}$ points to a closely half filled manifold. From these numbers two remarks arise $: i$. The larger $t_{2 \mathrm{~g}}$ population and moment are translated by large and localized DOS whereas $e_{\mathrm{g}}$ population is smeared out in rather broad and itinerant states [see 19]. ii. From the fact that a formally high spin (HS) configuration of Fe(IV) would give $M\left(t_{2 g}\right)=3 \mu_{B}\left(3\right.$ é) and $M\left(e_{g}\right)=1 \mu_{B}\left(1\right.$ é) and that a low spin (LS) one $M\left(t_{2 g}\right)=2 \mu_{B}$ ( 4 é) and $M\left(e_{g}\right)=0 \mu_{B}(0$ é), we notice that the calculated $t_{2 \mathrm{~g}}$ population and moment give an intermediate spin state between HS and LS configurations whereas the $\mathrm{e}_{\mathrm{g}}$ ones are far off. This suggests a larger covalency of the Fe-O bond involving $\mathrm{e}_{\mathrm{g}}$ states. With a back transfer Fe $\leftarrow \mathrm{O}$ through these states and the $\sigma$ bonds, the low oxidation state of closely -1 for oxygen and its magnetic moment can be explained (point $i$.). The energy difference between the F and NM configurations expectedly favors the former. This result is no more conforting when the ground state energies of the two AF configurations are compared with the NM one! Consequently the order of increasing stability of the ground state is : NM $\rightarrow \mathrm{AF}<111>\rightarrow \mathrm{AF}<001>\rightarrow \mathrm{F}$. The fact that we obtain a ferromagnetic ground state for $\mathrm{SrFeO}_{3}$ is in apparent contradiction with experiment. However the energy difference between $\mathrm{F}$ and $\mathrm{AF}<001>$ is only $5 \mathrm{mRyd}$, which would mean that the two magnetic configurations are close. Ferromagnetism actually appears when small amounts of $\mathrm{Co}$ substitute for $\mathrm{Fe}$. Moreover its neighboring homologue $\mathrm{SrCoO}_{3}$ with one more valence electron is ferromagnetic. Lastly, the magnitude of the $q$ vector is low $(0.1122 \pi / \mathrm{a} \sqrt{3})$ so that the helix is more similar to a ferromagnet. As to why the $\mathrm{AF}<001>$ configuration is more stable than the $\mathrm{AF}<111>$, it should be pointed that these configurations correspond to collinear and non-collinear AF's. Thus in the absence of the full energy versus q vector curve confrontations of energies are irrelevant. A thorough investigation of this and other magnetic perovskite oxides is underway.

\begin{abstract}
4. Prospective conclusions.
The calculations within the DFT/LSDA allow to extract accurate information on the electronic and magnetic structures of several types of compounds. This has been illustrated for two simple oxide systems with interesting magnetic properties. More complex magnetic systems can be treated as well. Although the ionic picture expected by the solid state chemist is not obtained, alternative representations of the magnetic structures and of the chemical bond are accessible. The latter is now made more quantitative through the COOP (crystal orbital overlap population) obtained by modulating the DOS with the sign and magnitude of the overlap integral. We recently implemented the COOP [20] in ASW method. From this a character to the chemical bond between two species can be assigned. Lastly the possibilities of such first principles self-consistent calculations as a predictive tool for the materials scientist in the search of new magnetic systems [21] can be underlined.
\end{abstract}

\title{
5. Ackowledgments.
}

I wish to thank Dr Michael Uhl of the Technical University of Darmstadt (Germany) -now on leave at the ICMCB-CNRS- for critical reading of the manuscript and fruitful discussions.

\section{References.}

[1]. P. Hohenberg and W. Kohn, Phys. Rev, 136 B864 (1964).

[2]. W. Kohn and L.J. Sham, Phys. Rev. 140, Al133 (1965).

[3]. R. Jones and O. Gunnarsson. Review of Modern Physics 161, 689 (1989).

[4].L. Hedin and B.I. Lundqvist. J. Phys. C4, 2064 (1971).

[5]. U. von Barth and L. Hedin, J. Phys. C5, 1629 (1972).

[6]. A.R. Williams, J. Küblerand C.D. Gelatt Jr., Phys; Rev. B19, 6094 (1979).

[7]. P. Blaha, K. Schwarz, P. Sorantin and S.B. Trickey. Coput. Phys. Commun. 59, 399 (1990).

[8]. J.P. Perdew and Y. Wang. Phys. Rev. B45, 13244 (1992).

[9]. P. Dufek, P. Blaha, V. Sliwko and K. Schwarz. Phys. Rev. B49, 10170 (1994)

[10]. M. Kamal, Ph.D. Thesis. Université de Metz, 1995.

[11]. J.F. Janak. Phys. Rev, B16, 255 (1977).

[12]. E.C. Stoner. Proc. Royal Soc. London, 165, 372 (1938).

[13]. J. Kübler and V. Eyert. « Electronic Structure Calculations » Vol. 3A : Ed. Buschow. VCH Verlag, Weinheim 1992.

[14]. P. Maestro, G. Demazeau, M. Pouchard, Th. Plante and P. Hagenmuller.Mat. Res. Bull. 16, 697 (1978).

[15]. J.B. Goodenough. « Les oxydes des métaux de transition », Eds. Gauthier-Villars, Paris (1973).

[16]. S. F. Matar, G. Demazeau, J. Sticht, V. Eyert and J. Kübler, J. de Physique France 2, 315 (1992).

[17]. H. Brändle, D. Weller, J.C. Scott, J. Sticht, P.M. Oppeneer and G. Güntherodt. Int. J. Mod. Phys. B7,345 (1993).

[18]. H. Oda, Y. Yamaguchi, H. Takei and H. Watanabe. J. Phys. Soc. Japan, 42, 101 (1977).

[19]. S. F. Matar, G. Demazeau, P. Mohn, V. Eyert and S. Najm. Eur. J. Solid State Inorg. Chem., 31, 615 (1994).

[20]. V. Eyert and S.F. Matar, implementation of the COOP in ASW code to be published in 1997.

[21]. G. Demazeau, B. Siberchicot, S. F. Matar, C. Gayet and A. Largeteau. J. Appl. Phys. 75, 4617 (1994). 